\title{
Smart Robotic Rover Enhancement in Safety Monitoring
}

\author{
Nurul Aqilah Herman ${ }^{1}$, Idris Mat Sahat ${ }^{2}$, Waheb Abdul Jabbar Shaif Abdullah ${ }^{1}$, MohdAzrul Hisham \\ Mohd Adib ${ }^{2}$ and Roshahliza M Ramli ${ }^{1}$ \\ ${ }^{1}$ Faculty of Electrical \& Electronics Engineering Technology, Universiti Malaysia Pahang, 26600 Pekan, Pahang, \\ Malaysia, nurulaqilahherman@gmail.com,waheb@ump.edu.my, roshahliza@ump.edu.my \\ ${ }^{2}$ Faculty of Mechanical \& Automotive Engineering Technology, Universiti Malaysia Pahang, 26600 Pekan, \\ Pahang, Malaysia, idriss@ump.edu.my, azrul@ump.edu.my
}

\begin{abstract}
Today in Malaysia, there is almost no available, affordable and portable liberating unmanned ground vehicle used in rescuing mission. Thus, this study focuses on enhancing a smart unmanned ground vehicle known as a robotic rover, to assist the human authorities during the hazardous condition. The present smart robotic rover has three main functions which are proposed to move robotic rover through obstacles surface, detect the leakage of dangerous gases and monitoring for collect information in a hazardous condition. The laboratory tests on this rover were successfully conducted and the results proved the ability of the rover. The rover will secure and safe the rescuer life rather than putting at the hazardous condition. Also, the rover gives a benefit to the endangered location to be secured faster and better. The advantage of this prototype rover is affordable and portable. This rover is simple and easy to handle. Hence, adequate to improve the rescuer skills in handling robotic rover in Malaysia.
\end{abstract}

Key words:Robotic, Unmanned Ground Vehicle, Safety \& Health, Rover

\section{INTRODUCTION}

Nowadays, an unmanned ground vehicle has vast and wide applications [1]-[4]in the real world. It can be implemented in scientific, space exploration, safety and weapon system endeavour. This type of vehicle goes where humans fear to tread, especially in hazardous and dangerous conditions. The unmanned ground vehicle or robotic rover system can be applied to assess, reduce or eliminate the risk. Through this, we can prevent any life-threatening or risk of death upon personnel when encountering such a scenario.

In the United State, NASA has built up the rocker-bogie suspension framework[4]-[6]for their rover and was executed in the Mars Pathfinder's Sojourner wanderer. The rocker-bogie suspension framework latently keeps every one of the six wheels on the robot in contact with the ground even on lopsided surfaces. This makes extraordinary traction. The rocker-bogie suspension instrument which was at present
NASA's affirmed structure for wheeled versatile robots, mostly in light of the fact that it had sturdy or flexible abilities to manage snags [7]-[9] and on the grounds that it consistently disperses the payload over its 6 wheels. It likewise can be utilized for different purposes to work in unpleasant streets and to climb the means. It was having loads of focal points yet one of the significant burdens is the turn of the instrument when and where is required [10]. The turn can be conceivable by giving individual engines to singular wheels which cause emerge in cost and complicacy in structure [11], [12].

In Malaysia, it is hard to find a robotic rover which the only way to get it is to buy from overseas which will cause us high cost with high transportation fees. Besides, generally robotic rovers nowadays which have not had a specific function such as gas detection, ability in climbing through obstacles and vision system. Aliff et al. [13]stated not only that, let firemen go into a place with a high risk of danger is not a wise decision. The robotic rover is considered to have a high level of versatility in the regular landscape if it can surmount impediments that are enormous in contrast with the size of its wheels. A rover must have enough traction from its back wheels to push the front wheels against an obstruction with enough power so they can move it up. Donahue et al., [14]stated that normally, a four-wheeled rover cannot climb snags bigger than a wheel sweep on the grounds that the back wheels need more traction. Sharukh et al. [15]stated that without traction, the wheels will slip and there will not be enough forward pushed to keep the front wheels in contact with the impediment.

Therefore, in this paper, the smart robotic rover well developed which is intended to move robotic rover through obstacles surface, detect the leakage of dangerous gases and monitoring for collect information in a hazardous condition.

\section{MATERIALS AND METHODS}

This section explains the methodology involved in developing the robotic rover from its design until its working principles.

\subsection{Design and development}

The robotic rover is structured utilizing the SolidWorks software. Each segment and parts of the item structure and drawing with the exact measurement as the genuine item. The segments and parts that are required to create are 
unmistakably attracted detail. In the SolidWorks, all parts are mated firmly together to form the design of the prototype. The orthographic and isometric perspective on the last design and the full component of the robotic rover shown in Figure 1.
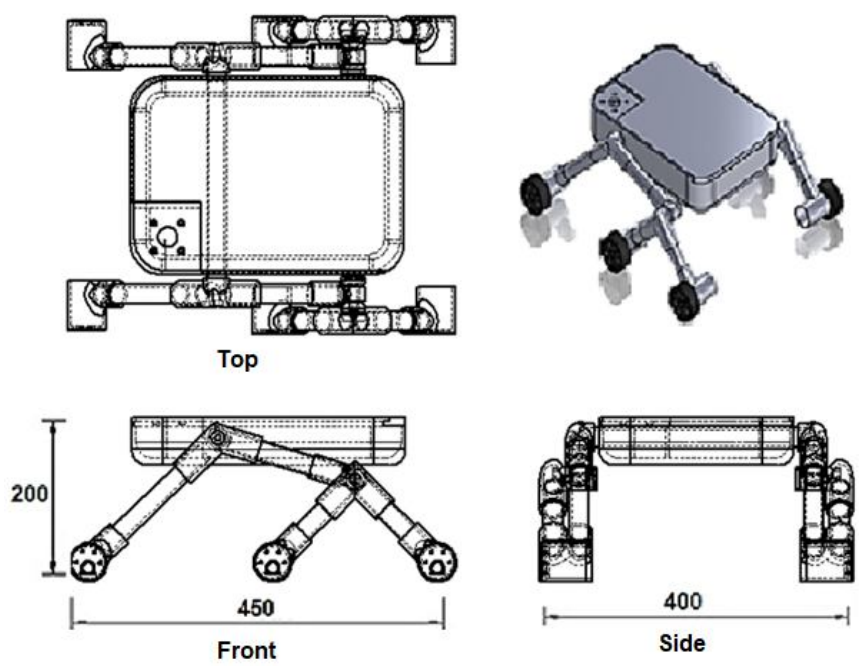

Figure1: Design and development of robotic rover with dimension unit millimeters.

The fabrication processes divided into two which are mechanical and electrical parts. In the mechanical part, the aluminium steel bar and polylactic acid (PLA) is selected as the main component. The aluminium steel is cut based on the dimension that already designs using a SolidWorks while the PLA is used on all connector on the rover.

The connector was designed using SolidWorks then transfer it to CURA software. CURA software used in order to fabricate all the connector by using a 3D printing machine and required to adjust the thickness and infill to get the better final product from 3D printing. After finish printed, the connector was assembled together with the aluminium as a chassis part.

For the electrical components as a controller, DC motor and Lippo battery were used as the power supply in order to move the robotic rover. An Arduino-Mega is performed as a controller to code and executes the requested command in the system. The coding is done integrated together with the Wi-Fi camera and gas sensor in order to perform the multifunctional robotic rover. The workflow of the robotic rover design and development process shown in Figure 2.

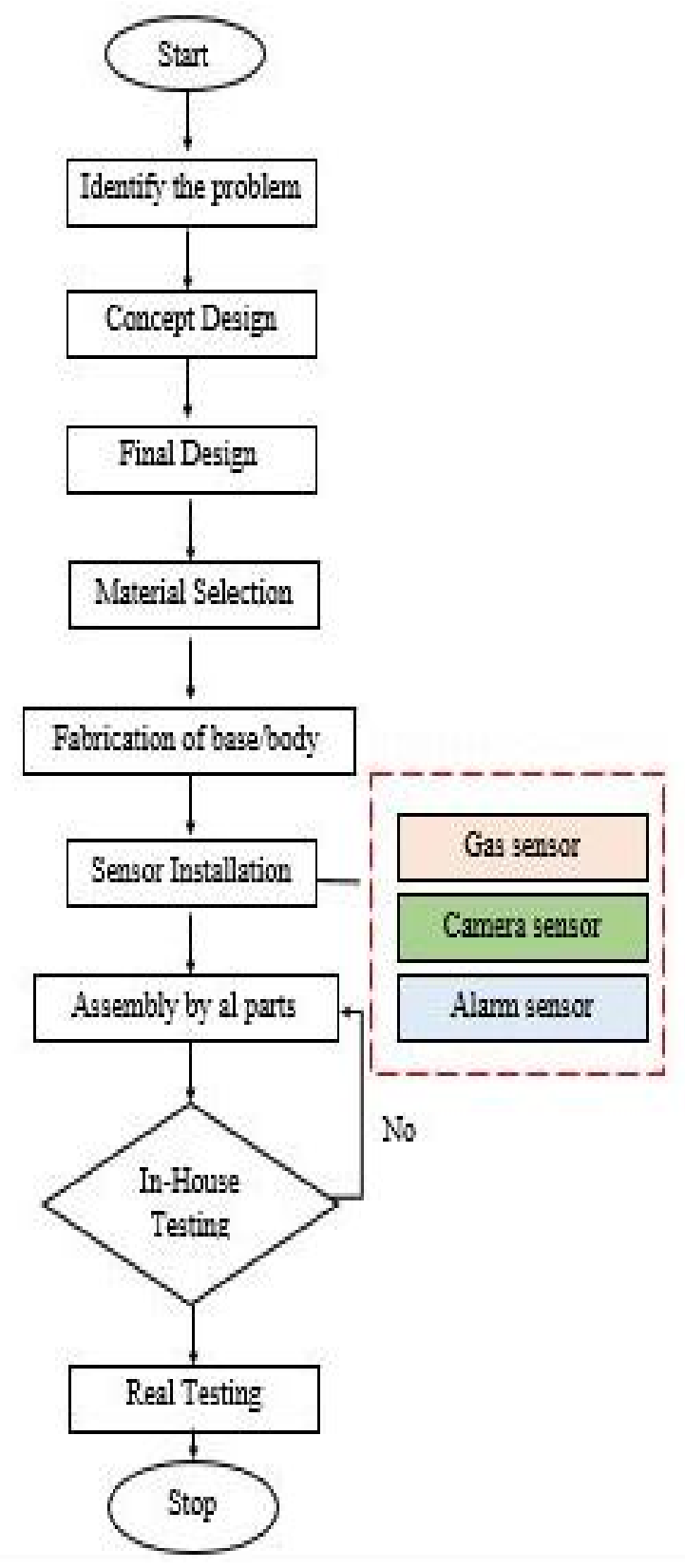

Figure 2: The workflow of the robotic rover development process

In addition, the design and the flexibility of locomotion, as well as the speed of the robotic rover, also need to be considered in order to finalize the final design of robotic rover.

Finally, after finish fabricates the mechanical and electrical parts, the assembly process will accomplish in order to present the robotic rover with multifunction. These smart robotic rover functions can move through the obstacle with the rocker-bogie mechanism, can get the real surrounding visual by the Wi-Fi camera and performed the gas sensor that can detect LPG gas and smoke. All functions were tested, and the video was recorded. 


\subsection{Materials and specifications}

In the material selection, the polylactic acid (SUNLU PLA) filament is used in this prototype development to create a complete structure of robotic rover. The material specification used in this study shown in Table 1.

Table 1: Material specification

\begin{tabular}{ll}
\hline Material & SUNLU PLA \\
\hline Print Temperature $\left[{ }^{\circ} \mathrm{C}\right]$ & $190-220$ \\
Length $[\mathrm{mm}]$ & 330 \\
Diameter $[\mathrm{mm}]$ & $1.75 \pm 0.05$ \\
Recommended printing speed $[\mathrm{mm} / \mathrm{s}]$ & $20 / 60$ \\
\hline
\end{tabular}

\subsection{Operation and Working Principles}

In this present robotic rover, the power supply unit provides $12 \mathrm{~V}$ DC supply to the processing unit. The two wheels in the front and rear sides are equipped with individual steering motors which allow the vehicle to turn into positions. Each of the six wheels on the rover has their own independent DC motor integrated with Arduino Mega and powered up by Lipo battery shown in Figure 3.

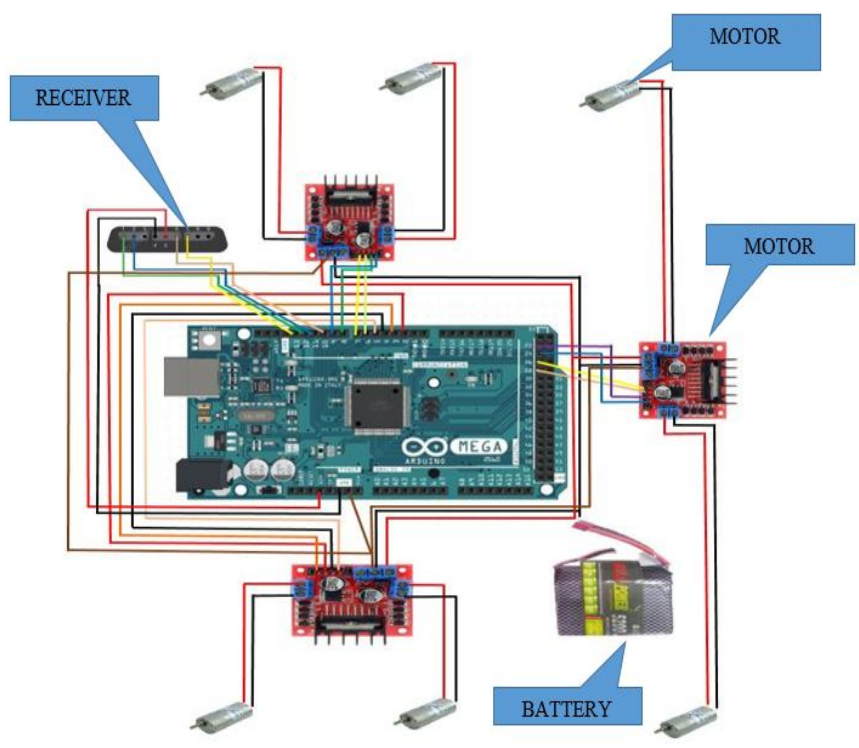

Figure 3: The integration of microprocessor and electrical components

Integration between Raspberry Pi and gas sensor (MQ-2) inside the smart robotic rover is well being operated shown in Figure 4.

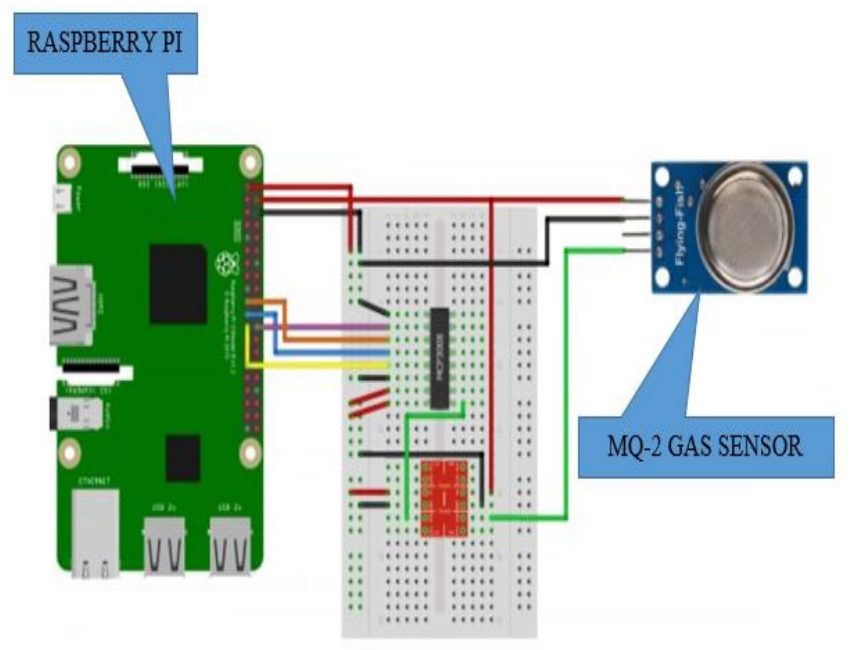

Figure 4: The integration of microprocessor and electrical components

\section{RESULTS AND DISCUSSIONS}

This section discussed the stress and displacement analyses on the design of the rover, its characteristics, the validity of its functions and the perceptions of the users as the results for this study.

\subsection{Stress and displacement analysis}

Figure 5 shows the analysis results of stress and displacement on the present design of robotic rover. From the results, the maximum stress obtained at body part with $100 \mathrm{~Pa}$. We aspect the heavy electrical components in the body parts affected these stress values. In the displacement analysis, the maximum displacement occurs at the front of the body with $15 \mathrm{~mm}$. This is also caused by the electrical component inside of the body itself. Besides, because of the rocker-bogie system used on this product, the system will have a free play on the front of the body as it goes through obstacles. When the rocker-bogie system goes through high obstacles on the right side, the body will move up according to the level of the system on the right side and the left side stays on the ground level. Thus, the displacement of the body is high.

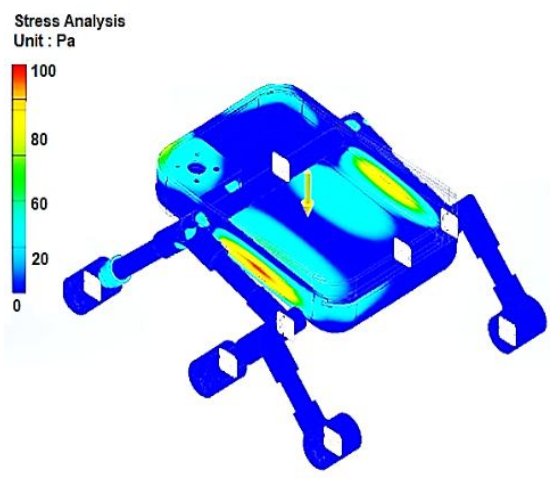

Figure 5 (a): Stress analysis 


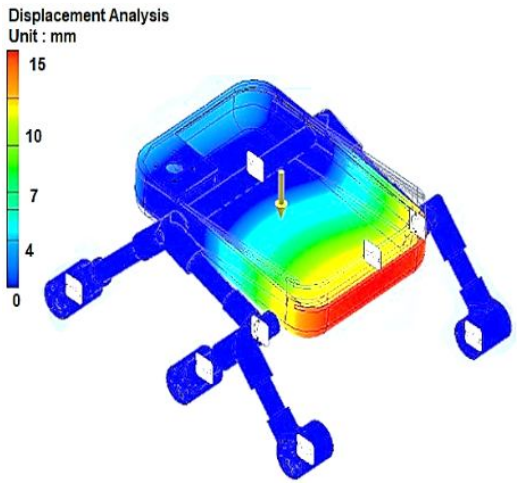

Figure 5 (b): Displacement analysis

\subsection{Smart robotic rover characteristics}

In this study, the prototype of the smart robotic rover is well developed. The rover mainly focuses on three functions. There are monitoring the hazard area condition, climb obstacles surface and detect the dangerous gases in exposure location. Figure 6 shows the main parts of the robotic rover. In the body part, we keep all the electrical components and controller of the rover system. The chassis was developed using an aluminium hollow tube in order to control the strength of the rover.

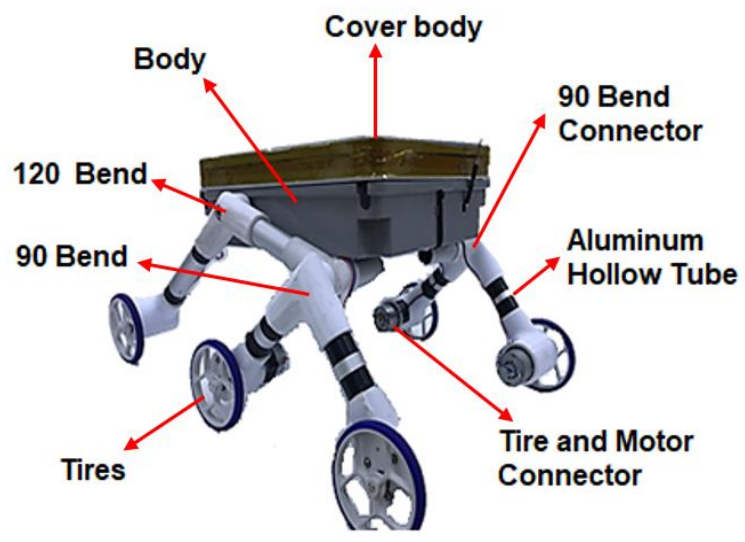

Figure 6 (a): Component used in the robotic rover

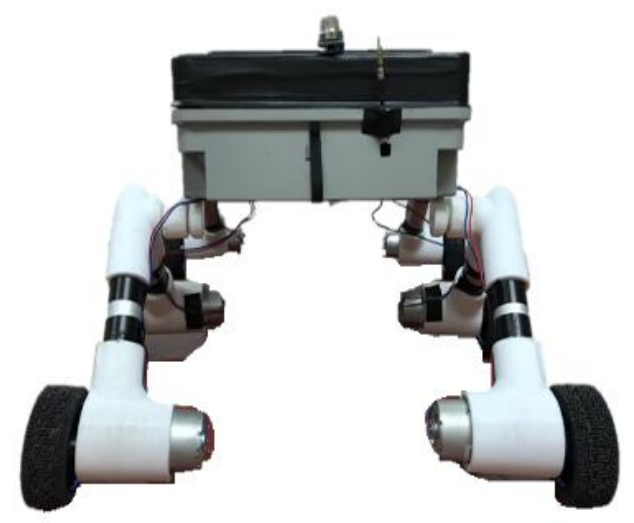

Figure 6 (b): Front side of the developed rover

\subsection{Functionality validation}

To validate the functionality of the proposed robotic rover the first prototype has been manufactured as presented in Figure 6. Preliminary investigation has been completed focusing on evaluating the proper function use of the designed robotic rover. Each wheel also has cleats, which provide the traction to crawl in soft sand and jump over rocks. The quasi-static force analysis can be done with the rover pose determined. The quasi-static distribution of force is used to determine whether the rover wheels will slip if the rover falls or tips over. It can also be used to calculate the energy consumption and if any actuator is close to saturation.

The result shows this present robotic rover is a good function. In Figure 7, 8 and 9, we clearly demonstrated the in-house testing on the prototype of a robotic rover. There are some limitations that we must face such as in terms of speed. This mechanism is unable to traverse at high speed due to the motors need to be balanced when lifting the vehicle's mass. Besides that, the selection of the wheels needs to be correct as well as it will affect the distribution of load on the wheels. Thus, the rover will tolerate a tilt of at least 45 degrees in any direction without overturning, depending on the centre of mass. Three tests were conducted at the laboratory, to monitor the hazardous location, climb obstacles surface and gas detector.

In the monitoring system, the rover used Wi-Fi connected with optical camera in order to observe the hazard location. However, this Wi-Fi only can detect in a small radius area around 50 meters. The images from the camera can be viewed on mobile apps, thus making the robotic rover become more user-friendly in monitoring the condition of the surrounding. The condition captured by the optical camera and transmitted via Wi-Fi shown in Figure 7.

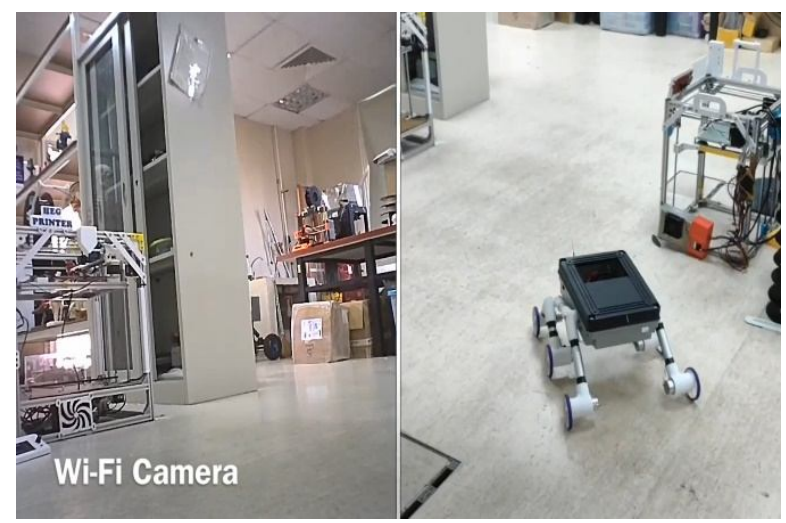

Figure 7: Validation test on the robotic rover to do monitoring by Wi-Fi camera installation

In the second test, we observed the function of the robotic rover to climb the surface of the obstacle. This robotic rover presented the good movement when climb the stair and stable in the obstacles surface as shown in Figure 8. Based on the experimentation results, the robotic rover was able to climb 10 stairs with a dimension of $10.16 \mathrm{~cm}$ by $10.16 \mathrm{~cm}$. Besides that, there are a few conditions for the robotic rover can be deployed such as before any accident happens for 
monitoring or surveillance processes and dangerous gas detection.

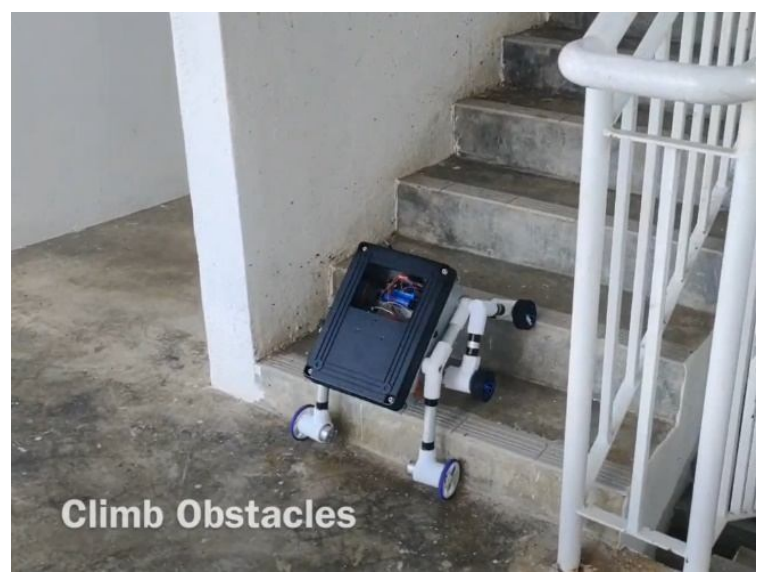

Figure 8: Validation test on the robotic rover to climb the stairs

Finally, in the third test, we implemented the gas detector in order to monitor the dangerous gases in the hazard area. Figure 9 shows the result of the MQ-2 gas sensor that produces the app. Cayenne. The app. automatic connected by mobile phone. The user should install the app. first and connect to the receiver from the gas sensor. This result shows that the MQ-2 gas sensor detected LPG gas, smoke, and CO. The value for LPG gas, smoke and Carbon Monoxide (CO) are 447.8, 2525.3 and 19290.2 respectively. All values of these compound are very high and if these gases are detected in a residential area, it could be considered that the place is in a dangerous condition and high risk.

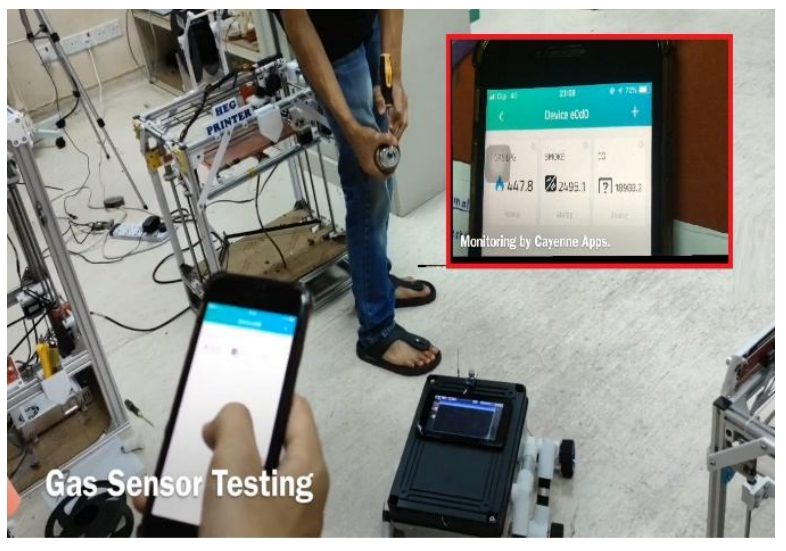

Figure 9: Validation test on the robotic rover to detect gas

\subsection{Response from user's perspectives}

The preliminary study on the present smart robotic rover observed that about $30 \%$ responses out of 30 respondents showed price will become the main priority in the potential users' perspective. Then, followed by function, easy handling, portable and weight are about $20 \%, 15 \%, 10 \%$, and $8 \%$ respectively. However, the brand and size of the device are the same about 9\% as shown in Figure 10. The users gave a good response and feel safety when handling this present rover. No negative feedback or complained during handling this rover is attained.

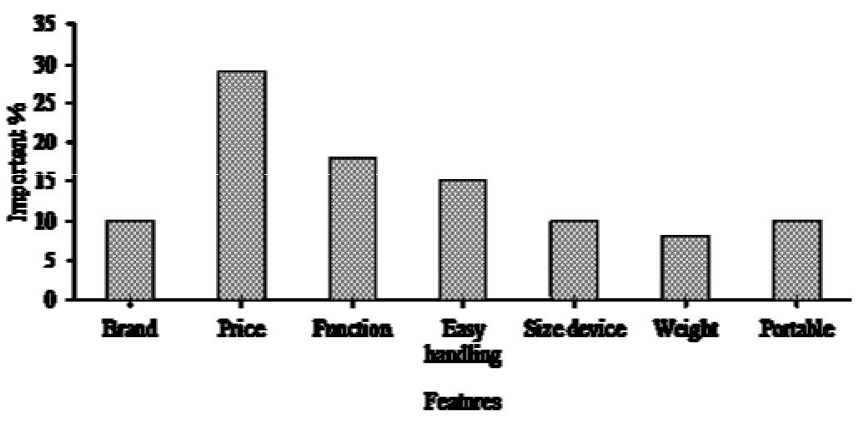

Figure 10: Preliminary survey on features of the robotic rover from users' perspectives

\section{CONCLUSION}

The new time of advances is being produced each day to upgrade the living nature of human life, which makes this study a proper and significant development to humankind. As a summary, in this study, the prototype of the smart robotic rover was successfully designed and developed. This robotic rover was capable to work in hazardous condition around the housing area as requested by the Malaysian Fire and Rescue Department. The rover is remote control operated and proficient of going through obstacles surface, scanning surrounding area with Wi-Fi camera and detecting non-corrosive poisonous gas and smoke where would reduce the risk of losing the rescuer life during rescuing. Nonetheless, there are numerous upgrades that can be made towards this unmanned ground vehicle for more prominent execution later on. Next, the authors intend to improve the design and usefulness of the rover, exclusively for the activities in operation and safety aspects.

\section{ACKNOWLEDGEMENT}

This project was funded by the Automotive Engineering Centre (AEC), University Malaysia Pahang under grant RDU1903103 and supported by the Medical Engineering \& Health Intervention Team (MedEHiT), Faculty of Mechanical \& Automotive Engineering Technology, Universiti Malaysia Pahang. The authors of this paper also would like to express their gratitude to Ahmad Ikhwan Hakim Ahmad Rufli, Khairul Rizal Bakri, Yong Pong Yew and Nasruddin Amir for supporting these research activities.

\section{REFERENCES}

1. P. Tokekar, J. Vander Hook, D. Mulla, and V. Isler, Sensor planning for a symbiotic UAV and UGV system for precision agriculture, IEEE Transactions on Robotics, vol. 32, no. 6, pp. 1498-1511, 2016.

2. S. Sheshrao Gajghate, D. S. Chinchkar, S. S. Gajghate, R. N. Panchal, R. M. Shetenawar, and P. S. Mulik, Design of Rocker Bogie Mechanism, vol. 4, no. $1,2017$. 
3. K. H. Chandu, P. H. Narayana, K. C. Teja, B. Sai, and Y. M. Mohan, Design and Fabrication of Rocker Bogie Mechanism, International Journal Scientific Engineering Technology Research, vol. 7, pp. 781-784, 2018.

4. G. Rabideau and E. Benowitz, Prototyping an onboard scheduler for the mars 2020 rover, 2017.

5. S. Ghosh, K. Otsu, and M. Ono, Probabilistic kinematic state estimation for motion planning of planetary rovers, in 2018 IEEE/RSJ International Conference on Intelligent Robots and Systems (IROS), pp. 5148-5154, 2018.

6. K. Otsu, G. Matheron, S. Ghosh, O. Toupet, and M. Ono, Fast approximate clearance evaluation for rovers with articulated suspension systems, Journal of Field Robotics, vol. 37, no. 5, pp. 768-785, 2020.

7. G. Gil, G. Savino, S. Piantini, and M. Pierini, Motorcycles that see: Multifocal stereo vision sensor for advanced safety systems in tilting vehicles, Sensors, vol. 18, no. 1, p. 295, 2018.

8. J. A. Crisp, M. Adler, J. R. Matijevic, S. W. Squyres, R. E. Arvidson, and D. M. Kass, Mars exploration rover mission, Journal of Geophysical Research: Planets, vol. 108, no. E12, 2003.

9. S. Melendez, The rise of the robots: What the future holds for the world's armies, Fast Company (June 12, 2017).

10. M. J. Schuster et al., The LRU rover for autonomous planetary exploration and its success in the SpaceBotCamp challenge, in 2016 International Conference on Autonomous Robot Systems and Competitions (ICARSC), pp. 7-14, 2016.

11. M. Vigneshwaran, R. Siddharthaa, G. Vijay, and S. Pravin Kumar, Design of All Terrian Vehicle Using Rocker Bogie Mechanism, International Journal of Mechanical Engineering and Technology, vol. 10, no. 3, 2019.

12. B. AjayKumar, C. Prashanth, C. SandeepKumar, T. Narasimha, and V. Reddy, Review on Rover with Rocker-Bogie Linkage Mounted with Ultrasonic Sensor and Bluetooth Module with Solar Energy, 2019.

13. M. Aliff, M. Yusof, N. S. Sani, and A. Zainal, Development of fire fighting robot (QROB), Development, vol. 10, no. 1, 2019.

14. K. C. Donahue and J. P. S. Hunt, Using Rocker-Bogie Suspension System to Build R-BEAR: Rocker Bogie Earth explorAtion Rover, 2019 NCUR, 2019.

15. M. Sharukh, B. Karim, N. V Sajin, and J. Thomas, Intelligent Deployable Mini Rover from the Mars Rover for Deep Narrow Scientific Investigation, 2019. 